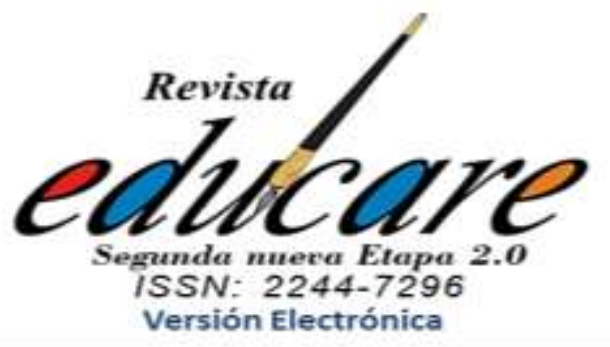

Volumen $25 \mathrm{~N}^{\circ} 1$ Enero - Abril 2021
(240-258)
Narváez Carrión Cesar Rafael *

ORCID: https:/orcid.org/0000-0002-2295-4206

Universidad Estatal de Bolívar

(Ecuador)

Larrea Astudillo Nelly Carolina***

ORCID: https://orcid.org/0000-0002-6663-3623

Cruz Báez Estafanía Cruz****

ORCID: https://orcid.org/0000-0002-3879-3611

Analuisa Maiguashca Jessica ****

ORCID: https://orcid.org/0000-0002-8413-4609

Universidad de las Fuerzas Armadas ESPE (Ecuador)
* Profesora de idiomas ( Departamento de Idiomas ) cnarvaez@ueb.edu.ec

**rcruz@espe.edu.ec

***jcanaluisa@espe.edu.ec

\section{ANÁLISIS EMPÍRICO SOBRE EL RENDIMIENTO ACADÉMICO DE LOS ESTUDIANTES EN LA ASIGNATURA DE INGLÉS CON EL USO DE VOSCREEN}

\author{
EMPIRICAL ANALYSIS OF THE ACADEMIC \\ PERFORMANCE OF THE ENGLISH STUDENTS \\ USING VOSCREEN
}

Recibido:

25-11-2020

Aceptado:

13-02-2021 

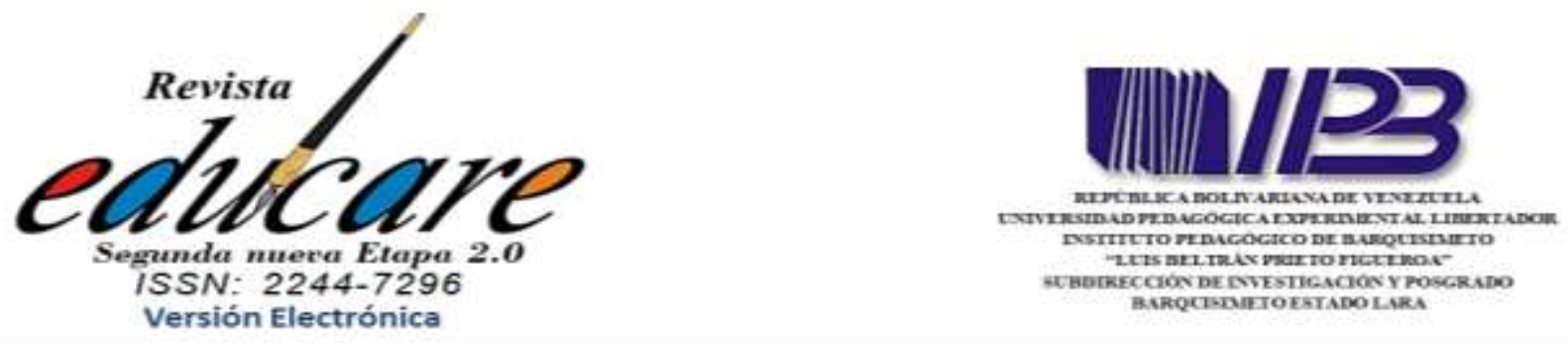

\title{
Resumen
}

La tecnología es parte importante de la sociedad actual y una interesante herramienta para mejorar el aprendizaje, su uso responde a la naturaleza lúdica que la caracteriza. El objetivo de la presente investigación es conocer si existe una correlación entre el uso frecuente de la aplicación VoScreen y el rendimiento académico de los estudiantes de idioma inglés de la Universidad Estatal de Bolívar, Ecuador. Esta investigación cuantitativa experimental, se basa en un muestreo no probabilístico intencional, aplicado durante un periodo de dieciséis semanas a un total de 270 estudiantes universitarios de diferentes edades y pertenecientes a diferentes carreras. Se analizó el uso o no de la aplicación VoScreen y los resultados obtenidos al final del periodo académico, constatando la investigación correlacional entre el uso de la aplicación y la obtención de mejores calificaciones al término del periodo académico. Finalmente, una encuesta aplicada reflejó la alta aceptación de la aplicación.

Palabras claves: Tecnologías de la información y la comunicación; rendimiento académico; aprendizaje del inglés

\begin{abstract}
Technology is an important part of the current society and an interesting tool for improving learning, its use responds to the ludic nature typical of them. The objective of the present research is to know if there is a correlation between the constant use of the VoScreen App and the academic performance of English language students in the Bolivar State University in Ecuador. This quantitative research is based on a convenience nonprobability sampling, applied during a sixteen-week period to a total two hundred and seventy university students with different ages and belonging to different colleges. It was analyzed the use or not of the VoScreen App and the obtained results of the term, confirming a correlation between the use of the application and obtaining of better scores at the end of the term. Finally, an applied poll reflected the high acceptance of the app.
\end{abstract}

Keywords: Information and communication technologies; academic performance; language learning. 


\section{Introducción}

Las tecnologías de información y comunicación han abierto el camino a nuevas estrategias para la educación en los distintos niveles académicos, áreas, sub áreas, cursos o programas y los diferentes actores son conscientes de ello. Tal es el caso de la enseñanza de idiomas extranjeros como el inglés, donde Carranza Alcántar et al. (2018), mencionan la percepción positiva en cuanto a la utilidad de la tecnología en su aprendizaje de un segundo idioma. Adicionalmente, Demir y Tavil (2021) constatan que los estudiantes prefieren materiales basados en la tecnología antes que materiales basados en texto para mejorar su comprensión oral, si bien los resultados obtenidos no mostraron una diferencia marcada en función de unos y otros materiales.

En este sentido, el desarrollo de tecnología ha dado pie a desarrollos metodológicos como el aprendizaje del idioma asistido por computador y el aprendizaje móvil de idiomas (CALL y MALL respectivamente, por sus siglas en inglés) en la línea de la llamada clase invertida en búsqueda de una mayor autonomía del estudiante y un incremento en su motivación (Forsythe, 2017). Para el óptimo aprovechamiento de dicha tecnología, se torna necesaria la constante actualización, como lo mencionan Curran et al. (2019), el uso creciente de las tecnologías digitales y móviles tiene importantes consecuencias en las políticas de organización, lugares de trabajo y la restricción en su uso y/o acceso puede entorpecer el desarrollo profesional, particularmente en la dinámica de esta clase invertida, de creciente aceptación en la educación superior (Trémion, 2019).

Dentro de esta metodología, una de las aplicaciones móviles recomendadas en el aprendizaje de idioma inglés, particularmente en los aspectos de comprensión oral y vocabulario es VoScreen (Tütüncu y Aksu, 2018; Alzatma y Khader, 2020; Boran et al., 2020; Türel y Davudova, 2019). Camilleri et al. (2016) profundizan en el uso de la herramienta, recomendando complementarla con actividades de producción oral y escrita, análisis fonético y diálogo.

Inmerso en el proceso educativo, un aporte importante es el de Tovar-Viera (2020) quien menciona que no es suficiente un conocimiento de lo tecnológico, lo pedagógico y del contenido en sí para un óptimo proceso de enseñanza, sino que es preciso articular estos tres aspectos para una práctica docente eficaz. 
Paker, (2012) señala que la enseñanza del idioma inglés no está enfocada a desarrollar la comunicación en los estudiantes, sino que planifican actividades dirigidas a la gramática y lecciones lo cual, minimiza el interés de los estudiantes, razón por la cual recomienda planificar actividades que involucren una comunicación verbal activa. Dehghanzadeh et al. (2019) rescatan lo prometedor del uso de la llamada gamificación para mejorar los procesos de enseñanza aprendizaje del idioma inglés, respaldando sus afirmaciones con la recolección de datos de 22 publicaciones entre 2008 y 2019. Molina-García et al. (2021) destacan de igual manera el valor de la gamificación para potenciar el aprendizaje del idioma inglés usando el juego para atraer la atención de los estudiantes, en vista de la alta importancia del idioma inglés en el contexto actual.

En este sentido, Alsawaier (2018) subraya el hecho de que algunos de los pilares sobre los cuales reposa la gamificación como el reforzar un cierto comportamiento o corregirlo por medio de la ausencia de recompensa o alguna forma de penalización son parte el eje mismo de la teoría conductista. Entre las principales motivaciones para jugar, Valderrama (2015) subraya, entre otras, la cooperación, la autonomía y la exploración, aspectos importantes a fomentar en la educación actual, mientras Ortíz-Colón et al. (2018) enfatizan el compromiso y la socialización como posibilidades de la gamificación a través de la interactividad y la interacción.

Por otro lado, la generación de nueva investigación en el área de la utilización de juegos para el proceso de enseñanza aprendizaje de idiomas extranjeros genera nueva información a la par del desarrollo mismo de la tecnología. Tal es el caso del estudio de Skykes (2018), quien menciona tres aspectos clave para profundizar el análisis del uso de los juegos para el aprendizaje: 1) el acceso aumentado a juegos basados en comunidades, 2) la incorporación significativa de la realidad virtual y 3) el acceso incrementado a juegos comerciales. Al margen de estos aspectos, cabe señalar la existencia de un sin número de herramientas tecnológicas que permiten mejorar las habilidades auditivas y la mayoría de estos recursos son gratuitos (Kiliçkaya, 2018).

Una de estas herramientas es VoScreen, que tiene como objetivo mejorar la pronunciación y habilidades de escucha de los usuarios mediante el uso de videos, ya que se conoce que mirar películas tiene beneficios, como incrementar la motivación en la enseñanza de idiomas y mejorar el vocabulario (İşcan, 2011). 
Es así que, el presente estudio pretende conocer si el uso de la herramienta tecnológica Voscreen influye en el rendimiento académico de los estudiantes de inglés en el departamento de idiomas de la Universidad Estatal de Bolívar. De igual manera, se pretende contribuir con estudios que permitan generar una evidencia empírica de la influencia de la app Voscreen en el proceso de enseñanza - aprendizaje del idioma inglés.

\section{Argumentación teórica}

Con la llegada la nueva era de la tecnología se ha visto la necesidad de modificar la manera de aprender, en el ámbito del aprendizaje de un idioma extranjero el uso de las aplicaciones sea móviles o web, adquieren de manera progresiva un rol importante en la educación, puesto que su creación tiene como objetivo en facilitar el acceso para la práctica de una segunda lengua, rompiendo esquemas ya sean físicas, temporales y materiales.

VoScreen una aplicación web y móvil utilizada en el aprendizaje de idiomas extranjeros, desarrollada para compensar la deficiencia de elementos de escucha y pronunciación. Taylan (2018) hace una descripción bastante detallada al respecto, al mencionar que la aplicación se basa en el uso de videos con una duración promedio de 15 segundos que varían en función de la categoría seleccionada por los estudiantes, quienes deben responder preguntas sobre lo observado. Las respuestas correctas se califican automáticamente, mientras que las incorrectas se eliminan de la puntuación total y se registran en el sistema. Dichos puntajes generan un sentido de competencia entre los usuarios. El autor menciona además que la aplicación permite archivar los fragmentos de su preferencia en listas de reproducción personalizadas. Además, Voscreen permite que los entrenadores o maestros puedan monitorear los resultados. Otro interesante componente es la posibilidad de enfocarse en determinadas estructuras del idioma o graduar la dificultad de los ejercicios de alguna manera a través de las funcionalidades VoStructure y VoStep, respectivamente.

Esta herramienta forma parte de las citadas por Yurdagül (2018) en su investigación sobre el uso de teléfonos inteligentes para el aprendizaje del idioma inglés, en el grupo de sitios/aplicaciones basados en video. Es importante subrayar que la aplicación puede articularse sin mayores dificultades a la sugerencia más reportada en dicho estudio en cuanto a su diseño, la práctica de vocabulario. 
Sin embargo, a pesar de los beneficios en cuanto a motivación y dinamismo como resultado del uso de la tecnología en prácticas ligadas a la educación, a través de dispositivos móviles, varios estudios mencionan efectos negativos en el desempeño académico a causa de la dependencia hacia los teléfonos inteligentes (Samaha y Hawi, 2016; Alkhunzain, 2019). De acuerdo a Erenli (2013) esto puede generar el aislamiento social por lo que es necesario evitar al uso excesivo para evadir la desviación del propósito educativo en el aprendizaje de los estudiantes.

Adicionalmente, se registran las consideraciones de Subrahmanyam et al. (2013) quienes evidencian un impacto del hábito de efectuar multitareas y la comprensión lectora, señalando un impacto negativo tanto en el caso de la lectura en papel como de la lectura en dispositivos electrónicos, con una incidencia mayor en el segundo caso. En este sentido, Yienger (2016) menciona una incidencia en la retención de información a causa de la lectura en dispositivos electrónicos en niños pequeños.

De una manera más general, la incorporación del uso de teléfonos inteligentes para el aprendizaje de idiomas extranjeros es un aspecto que posee eventuales limitantes como por ejemplo, las dificultades para la lectura al tratarse de una pantalla de tamaño reducido, problemas técnicos en el acceso a internet, fallas en los componentes físicos del equipo y problemas en los diferentes programas, sin mencionar la distracción de los estudiantes dentro del aula de clase (Chartrand, 2016).

Adicionalmente, como una parte preocupante del desarrollo tecnológico es importante mencionar la presencia de varias amenazas que han surgido a través de internet, al verse expuestos a contenido inadecuado para su edad. Estos riesgos de abuso tienen indudablemente implicaciones en el plano educativo (Machimbareena et al., 2018). Por otro lado, existe una desventaja adicional si se considera la existencia de la publicidad online cuya presencia constante puede resultar en un sentimiento de intrusión y una interrupción de los procesos cognitivos como lo reportan Lewandowska y Jankowski (2017).

De acuerdo a Taylan (2018) en la enseñanza de un idioma extranjero, es importante desarrollar la habilidad de hablar y que el estudiante se pueda desenvolver en una conversación de forma adecuada. Este proceso se puede fortalecer mediante la herramienta Voscreen, que permite al estudiante escuchar fragmentos de audios, en contextos reales, donde la pronunciación y el vocabulario son expuestos por hablantes nativos y no nativos de 
diferentes rincones del planeta. Adicionalmente, el diseño de la aplicación permite brindar claves visuales para facilitar la tarea, si bien lo reducido de los videos contribuye a centrar el interés en la destreza de escuchar, con la ventaja de poder reproducir el material tantas veces como les sea necesario (Krumlová, 2017)

Alhawad y Almunif (2020) mencionan que los estudiantes están en la capacidad de alcanzar el objetivo de hablar fluidamente el idioma inglés, por otro lado, Kiliçkaya (2018) especifica la práctica fuera del aula de clase como una alternativa complementaria al uso del inglés como lengua de instrucción, a fin de garantizar las aportaciones y llegar al reconocimiento de elementos fonológicos fundamental para lograr una comunicación oral eficiente. Erkan (2020) por su parte, alude la capacitación formal en materia de tecnologías de la información y la comunicación como un aspecto de eventual impacto en el estilo de enseñanza de los docentes contribuyendo a sus competencias en la enseñanza.

Demírbílek y Yücel (2011), aluden al hecho de que los estudiantes han sido sustraídos de la educación individualizada debido a que existe aglomeración en las aulas. La enseñanza está enfocada sólo en exámenes y los recursos educativos consisten sólo en utilizar libros. Si el objetivo de la educación de idiomas extranjeros consiste en que los estudiantes hablen y entiendan, el sistema educativo necesita integrar herramientas tecnológicas. Estas herramientas incluyen varias aplicaciones web en especializadas en el aprendizaje en lenguas extranjeras. En el caso específico se las aplicaciones móviles, se reportan resultados más eficientes y una mayor motivación (Ekinci y Ekinci, 2017).

García, (2013) en un estudio de investigación sobre la aplicación interactiva Duolingo en la enseñanza de inglés, concluyó que la interpretación se puede considerar como un aporte importante dentro del desarrollo del idioma en los estudiantes. Vesselinov y Grego (2012) en su estudio sobre la aplicación Duolingo, aplicado a 386 estudiantes, previo al desarrollo de la evaluación final. Los alumnos practicaron en la aplicación antes de rendir el examen. Esto generó un efecto positivo en los resultados. Los participantes señalaron que la aplicación ha apoyado el aprendizaje de idiomas extranjeros de forma amigable.

Al analizar Busuu, otra de las aplicaciones interactivas de amplia utilización para el aprendizaje de lenguas extranjeras, Vesselinov y Grego (2016) utilizan esta herramienta para la evaluación de una muestra de 196 participantes, durante la práctica y al final de una actividad. La aplicación de Busuu registró una contribución importante para mejorar la 
educación en lenguas extranjeras en un $84 \%$ de los participantes. El 74\% de los estudiantes mejoró su nivel de idioma extranjero, logrando un aumento de 12 puntos en las habilidades, los alumnos indicaron que la aplicación influyó positivamente en su aprendizaje y que el proceso de enseñanza se tornó interesante.

Adicionalmente, el citado estudio de İşcan, (2011) enfocado en VoScreen reveló que observar películas en la educación de idiomas extranjeros tiene muchos beneficios positivos, como incrementar la motivación en los estudiantes y mejorar el vocabulario. El autor menciona particularmente el modo en que la utilizan los maestros, así como sus efectos positivos en el aprendizaje de lenguas extranjeras. Bal (2019) por su parte destaca entre sus resultados la preferencia estudiantil de trabajar con videos de corta duración para el mejoramiento de su habilidad de comprensión oral.

En contraparte, es importante tomar en cuenta las limitantes de la aplicación entre las que se mencionan la falta de actividades de escritura, la ausencia de notificaciones, la necesidad de conexión a internet y un cierto nivel de estrés (Ekinci y Ekinci, 2017), limitantes a tomarse en cuenta para la complementación con otras estrategias o herramientas para el mejoramiento del desempeño estudiantil.

En cuanto al presente estudio, éste fue llevado a cabo en la Universidad Estatal de Bolívar, que posee como requisito previo para la graduación y obtención del título de tercer nivel, la aprobación de una lengua extranjera, tal como lo es el idioma inglés.

Cabe señalar que los estudiantes son personas adultas y cursan diferentes carreras. El historial de práctica de la aplicación en mención fue el instrumento que proporcionó la información sobre la práctica continua y se contrastó con los resultados obtenidos por los estudiantes al término del periodo académico.

\section{Metodología}

Durante el periodo académico abril - agosto 2020 se registró un total de 1974 estudiantes inscritos en la asignatura en mención, siendo tomada una muestra estadística por conveniencia de 270 estudiantes, que conforman los sujetos de estudio de la presente investigación.

Los 270 estudiantes fueron divididos en 5 aulas: 3 aulas del nivel A1 con un total de 187 estudiantes y 2 aulas en el nivel A2 con 83 estudiantes. Es así que, en cada aula se aplicó 
la App Voscreen con el objetivo de dar soporte y refuerzo a las actividades planificadas en la asignatura.

La aplicación de la herramienta se hizo con base al hecho de haber sobresalido en el concurso "Reimagine Education 2016", organizado por Wharton Business School, UPENN (EE. UU.), en donde se presentó más de 500 iniciativas, de las cuales Voscreen desarrollado en Turquía, fue seleccionado como tercero en la categoría "Mejor aplicación educativa", esto en razón de que superó los 1,7 millones de usuarios y se ha utilizado en más de 74 países. El acceso a la aplicación se da por medio de cuentas de Facebook o correos electrónicos (Taylan, 2018).

La frecuencia de utilización varía entre 4 y 7 veces por semana. La consigna dada a los estudiantes fue que practicarían 5 minutos diarios, de lunes a jueves (los días que tenían clases virtuales de idioma inglés), recomendándoles que lo realizaran durante más tiempo y con mayor frecuencia. En este punto, el formato lúdico de la aplicación contribuyó a que en ocasiones se llegara a superar los 60 minutos de práctica, lo cual se pudo constatar al monitorear los puntajes de los estudiantes. Adicionalmente, en el marco del desempeño estudiantil, se aplicó una encuesta, la cual fue respondida por el $65 \%$ de los estudiantes, donde se reportan las dificultades tecnológicas como uno de los factores predominantes para no utilizar la aplicación.

Una vez finalizado el tiempo establecido para la utilización de la App en cada aula, se obtuvo los datos por medio del reporte que genera Voscreen, donde se puede observar el número de intentos realizados por cada estudiante, el número de respuestas correctas e incorrectas y la puntuación obtenida tras cada participación. Además, se utilizó el promedio de calificaciones obtenidas por los 270 estudiantes al final del periodo académico. De tal manera que con el reporte y las calificaciones se pudo identificar a aquellos estudiantes que hicieron uso de la App y de igual forma a los que no, con el objetivo de dividirlos en dos grupos estadísticos diferentes para el estudio cuantitativo experimental.

Una vez detectados los dos grupos, quienes usaron la aplicación VoScreen y quienes no lo hicieron, se pudo realizar una comparación de los promedios entre dichos estudiantes, para posteriormente realizar un gráfico comparativo de las notas entre los dos grupos como se observa en la figura 1. 
Por otro lado, se realizó la prueba de hipótesis con medias y varianzas conocidas, utilizando la distribución normal z ya que se ajusta a la tendencia de los datos y al número de la muestra (Fallas, 2012). Para ello, se plantearon las siguientes hipótesis:

Nula, donde la media de las calificaciones con el uso de la App es menor o igual a la media de las calificaciones sin el uso de la App.

$$
H_{0}=\bar{X}_{A P P}-\bar{X}_{N O A P P} \leq 0
$$

Alternativa, en la que la media de las calificaciones con el uso de la App es mayor a la media de las calificaciones sin el uso de la App.

$$
H_{a}=\bar{X}_{A P P}>\bar{X}_{N O A P P}
$$

Para la presente investigación de aplicó la siguiente formula de la prueba de hipótesis

$$
z=\frac{\left(\overline{\bar{X}_{1}}-\bar{X}_{2}\right)-D_{0}}{\sqrt{\frac{\sigma_{1}^{2}}{n_{1}}}+\frac{\sigma_{2}^{2}}{n_{2}}}
$$

Reemplazando los valores, se obtuvo:

$$
z=\frac{7,8989-6,829}{\sqrt{\frac{0,52462}{188}}+\frac{4,6618}{88}}=\frac{1,0699}{0,489}=2,15
$$

Con el valor de $\mathrm{z}$ calculado se obtiene el valor $\mathrm{p}$ de las tablas de distribución normal.

Como probabilidad de que ocurra el evento se toma como referencia el valor p=0,9842, y para la comprobación de la hipótesis el valor $-\mathrm{p}: 1$ - 0,9842=0,0158

Como el valor $\mathrm{p}$ de la prueba de hipótesis es menor al nivel de significancia, se rechaza la hipótesis nula. Es decir, que el promedio de las calificaciones de los estudiantes que utilizaron la APP es mayor que aquellos que no.

\section{Resultados}

Como se mencionó anteriormente, con el objetivo de conocer si el uso de la App influye en el rendimiento académico de los estudiantes del idioma extranjero- inglés, se realizó una comparación entre dos grupos de estudio detectados que hacen las veces de grupo 
de grupo experimental y grupo de control: los estudiantes que utilizaron Voscreen y los estudiantes que no la utilizaron, donde se obtuvo como resultado lo siguiente:

\section{Figura 1.}

Comparación de notas obtenidas con y sin el uso de la App Voscreen.

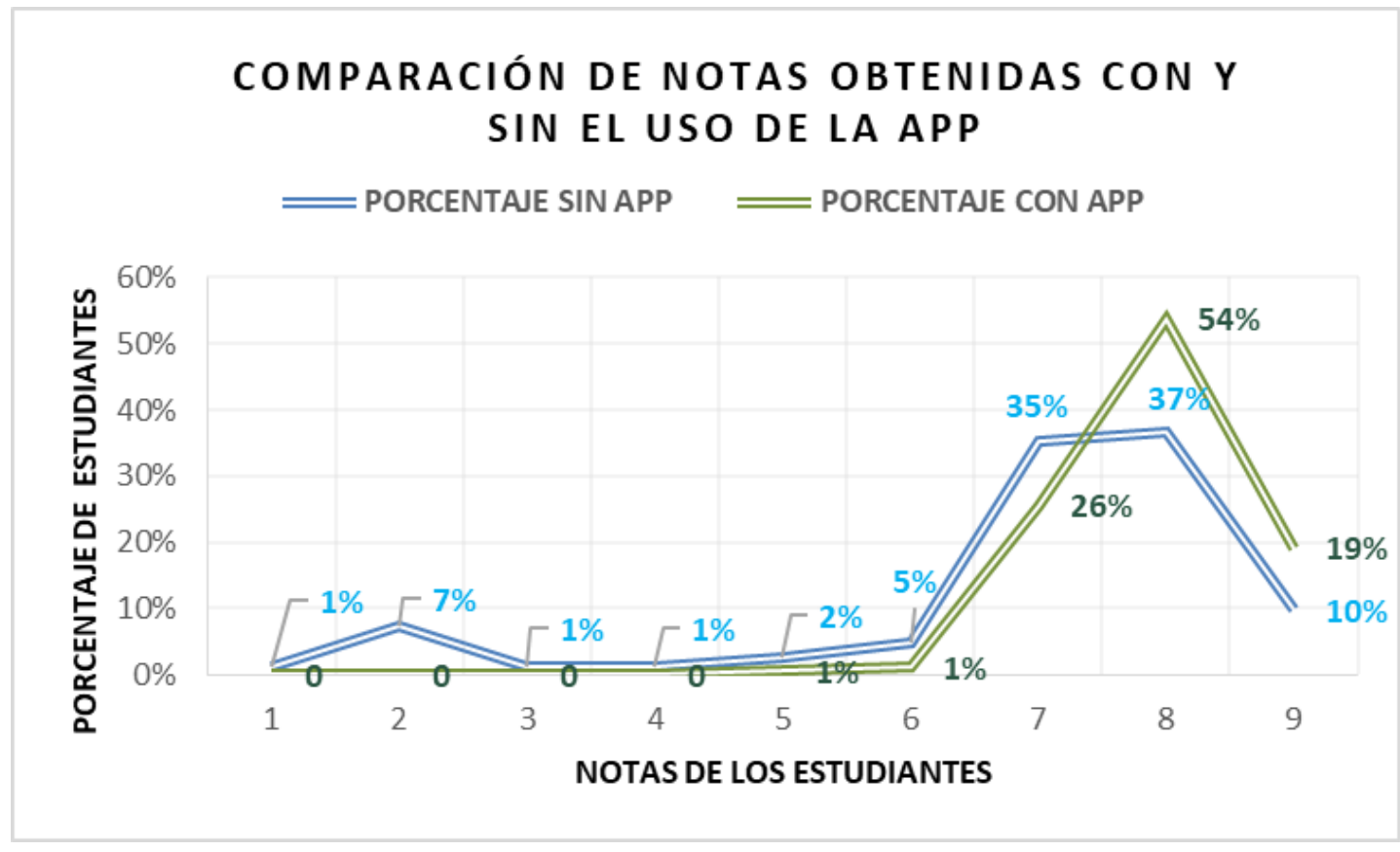

Fuente: Elaboración propia.

Se evidencia en la figura 1 que existe un incremento en las calificaciones de los estudiantes que utilizaron la APP ya que el $54 \%$ de ellos obtienen notas en torno a los 8 puntos. Mientras que, el $26 \%$ obtuvo una calificación de 7 sobre 10 y por último, el $19 \%$ obtuvo 9 puntos como promedio. Finalmente, se observa que el 1\% de los estudiantes obtuvieron calificaciones de 5 y 6 puntos. No se obtuvieron notas menores a 5 dentro del grupo de estudiantes que utilizaron la APP Voscreen.

Por otra parte, los estudiantes que no utilizaron la aplicación reflejan calificaciones menores ya que el $37 \%$ obtuvieron notas de 8 sobre 10, el $35 \% 7$ puntos y únicamente el 10\% refleja calificaciones de 9 puntos. De igual manera se refleja un $7 \%$ de estudiantes con notas bajas (2 puntos sobre 10) y de igual manera el $5 \%$ obtuvieron calificaciones de 6 puntos. A diferencia del grupo anterior, aquí se refleja la obtención de calificaciones menores a 5 puntos, ya que el 1\% obtuvieron calificaciones de 1, 3 y 4 puntos sobre 10 . 
Las muestras son diferentes, por lo que se realiza el gráfico considerando los porcentajes de calificaciones en vista de que se tiene dos poblaciones distintas, para un segundo análisis se realizó lo siguiente:

\begin{tabular}{|c|l|}
\hline \multicolumn{2}{|c|}{ Grupos estadísticos diferentes } \\
\hline $\begin{array}{c}\text { Prueba de } \\
\text { Hipótesis }\end{array}$ & \multicolumn{1}{|c|}{ Comprobación de } \\
hipótesis \\
\hline $\mathrm{p}=0,9842$ & 0,0158 \\
\hline \multicolumn{2}{|c|}{ valor - $\mathrm{p}: 1-0,9842=$} \\
valor -p obtenido se rechaza la hipótesis \\
nula.
\end{tabular}

Es decir que el promedio de las calificaciones de los estudiantes que utilizaron la APP es mayor que aquellos que no.

Finalmente, durante el periodo de clases, se observó un aumento en la motivación y en la práctica constante, fruto de la utilización periódica en la aplicación VoScreen. Dicha motivación se puede evidenciar en los resultados de la encuesta aplicada, como se indica a continuación:

Figura 2: Causas por las que los estudiantes no usan la App Voscreen.

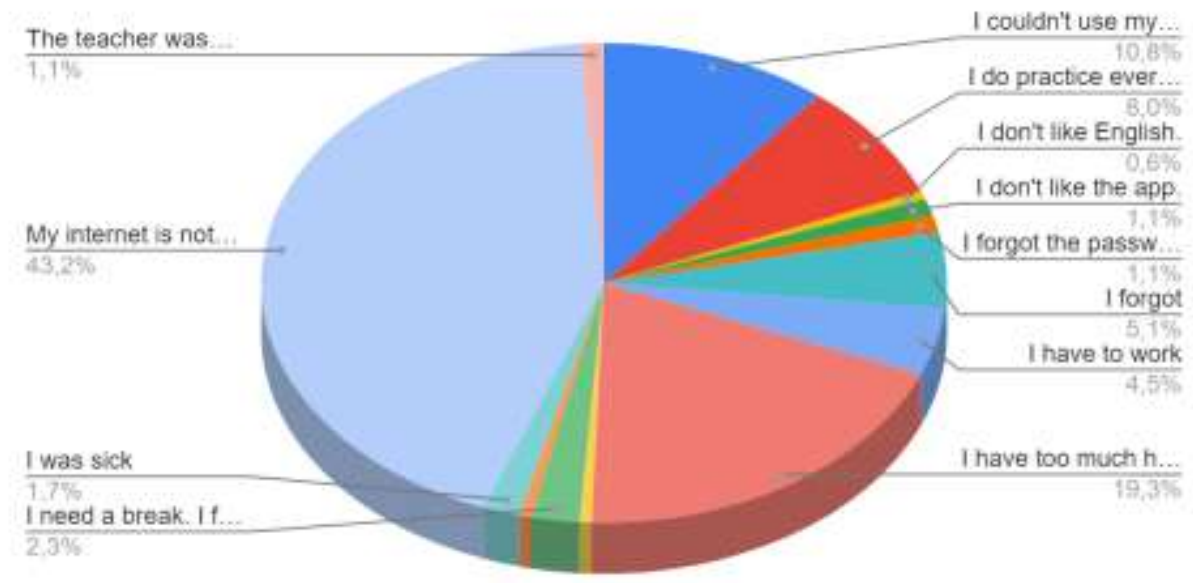

Fuente: Elaboración propia. 
Dado que los lectores no son necesariamente bilingües, sugiero describir los resultados en un párrafo en español y ampliar un poco el análisis. Se observan 12 respuestas distintas, en la figura 2, se puede apreciar que únicamente el 1,1\% reporta usar esta herramienta a disgusto mientras que solamente un $0,6 \%$ reporta no haber utilizado la App ya que el idioma inglés no es de su agrado. Además, reportan que la mayor causa para que no hayan practicado debidamente fue la calidad de su conexión a internet (43,2\%),

\section{Discusión}

Los resultados obtenidos que muestran la correlación entre el uso de la aplicación y los resultados en cuanto al aprendizaje del idioma sustentan la investigación de Karaduman (2018) que muestra su mejora en los aspectos de escucha y pronunciación, si bien el presente estudio lo muestra de una manera más global.

Asimismo, el entusiasmo durante el proceso de aprendizaje se pudo evidenciar con la encuesta final, lo cual se alinea con los resultados obtenidos por Taylan (2018). Por otro lado, cabe mencionar la utilidad de la naturaleza de la aplicación, ideal para la práctica autónoma como lo menciona Kiliçkaya (2018), con el objetivo de lograr una exposición constante al idioma-objetivo. Conclusiones todas que apoyan los resultados de la presente investigación.

En un sentido más amplio, Ozer \& Kılıç (2018) constataron mejoras en la adquisición del idioma inglés. A diferencia del presente estudio, los autores no se enfocan en la utilización exclusiva de la herramienta VoScreen sino en el uso de tecnología móvil, en una manera más general. Su estudio analiza dichas mejoras de forma integral, incluyendo las cuatro destrezas del idioma inglés, tal y como se ha hecho en el presente estudio, pues se ha considerado los resultados globales al final del periodo de clases.

En contextos más cercanos, son aportes importantes que respaldan el presente estudio, las apreciaciones de Bernal y Feyen (2017), valorando VoScreen como una aplicación que se constituye en un ejercicio de escucha, pronunciación, lectura y comprensión, particularmente al tratarse de otra institución de educación superior ecuatoriana, la Universidad de Cuenca. En esta misma línea, los resultados obtenidos respaldan la elección de Viera y Sánchez (2020), y Blanco Villamil (2018) quienes usan VoScreen como una de las herramientas para probar el impacto del conocimiento tecnológico, pedagógico y de contenido para elevar la efectividad de la enseñanza del idioma 
inglés en la Universidad de Cotopaxi (Ecuador) y el Colegio San Rafael IED de Bogotá (Colombia).

Cabe destacar que estos aportes así como el de la presente investigación respaldan la recomendación hecha por Ronquillo Perlaza (2016) al incluir Voscreen en una serie de recursos destinados a dinamizar el aprendizaje del idioma inglés luego de concluir en su investigación que uno de los principales inconvenientes en el proceso es el limitado acceso de los docentes y estudiantes a las tecnologías de la comunicación y la información.

\section{Conclusiones}

La aplicación VoScreen, basada en la gamificación y por ende en el modelo pedagógico conductista, es una herramienta útil para elevar la motivación en el proceso de aprendizaje de idioma inglés. La aplicación se puede usar un complemento valioso para complementar la exposición de los estudiantes al idioma meta. La evidencia del presente estudio demuestra su utilidad como espacio de práctica para mantener la práctica constante fuera del aula desde la perspectiva de la curiosidad y la motivación propia del estudiante con resultados de mejora significativa en el manejo del idioma inglés. Como implicaciones surgen interrogantes acerca del grado de impacto en áreas específicas del aprendizaje como el vocabulario, la comprensión y producción oral, o el conocimiento sintáctico gramatical. Por otro lado, es importante conocer el impacto del incremento en la utilización de la tecnología y de la gamificación en un cierto grado de adicción a la tecnología.

En el momento actual, un porcentaje significativo de estudiantes enfrentan problemas de conectividad en su proceso de aprendizaje que dificultan el acceso a las clases virtuales, un inconveniente que se puede paliar con el uso de la aplicación en mención, limitando así la cantidad de idioma a la cual dichos estudiantes se hallan expuestos. La Investigación adicional se hace necesaria para constatar en qué medida dicho inconveniente impacta en la calidad del aprendizaje. Por otro lado, la motivación estudiantil como resultado de la implementación de la aplicación VoScreen juega un rol importante en el aprendizaje de los estudiantes, como lo demuestran las estadísticas de aprobación al final del semestre en la muestra del estudio. No solamente se logra una motivación creciente hacia la práctica del idioma sino que se cambia al menos parcialmente los estereotipos hacia la lengua meta. Finalmente, la gratuidad del recurso en mención contribuye en gran medida a su implementación. 


\section{Referencias}

Alhawad, A., \& Almunif, S. (2020). The Effect of the Voscreen Application on Oral Performance Among Saudi Efl Learn. Route Educational and Social Science Journal, 7(52), 109-116. https://doi.org/10.17121/ressjournal.2751

Alkhunzain, A. S. (2019). An Empirical Study on Smartphone Addiction of the University Students. International Journal of Interactive Mobile Technologies (iJIM), 13(12), 184-195.

Alsawaier, R. S. (2018). The effect of gamification on motivation and engagement. International Journal of Information and Learning Technology, 35(1), 5679. https://doi.org/10.1108/IJILT-02-2017-0009

Alzatma, A. A., \& Khader, K. (2020). Using Mobile Apps to Improve English Speaking Skills of EFL Students at the Islamic University of Gaza نيسحتل تار اهم مادختسا تاقيبطت فتاهلا لومحملا.

Bal, S. (2019). The integration of ict tools into listening skill classes to improve listening comprehension of efl learners (Master's thesis, Bursa Uludağ Üniversitesi).

Bernal, M., \& Feyen, J. (2017). A preliminary response from the Faculty of Psychology students of the University of Cuenca to the modified EFL teaching approach. Maskana, 8(1), 61-69.

Blanco Villamil, A. K. (2018) Promover el fortalecimiento de las habilidades de lectura y escritura en inglés a través de historias cortas en un ambiente virtual de aprendizaje (AVA) basadas en valores en los estudiantes del grado quinto.

Boran, G. S., Çiloğlan, F., \& Durmaz, B. N. (2020). An Investigation of Foreign Language Proficiency of Cabin Crew Candidates during Employment Process. Language Teaching (TOJELT), 5(3), 131-145.

Camilleri, V., Dingli, A., \& Montebello, M. (2016). Gamify your classroom!A guidebook tool for an alternative pedagogical approach to class-based teaching.

Carranza Alcántar, M. D. R., Islas Torres, C., \& Maciel Gómez, M. L. (2018). Percepción de los estudiantes respecto del uso de las TIC y el aprendizaje del idioma inglés. Apertura (Guadalajara, Jal.), 10(2), 50-63.

Chartrand, R. (2016). Advantages and disadvantages of using mobile devices 
in a university language classroom. Bulletin of the Institute of Foreign Language Education Kurume University, 23, 1-13.

Curran, V., Gustafson, D. L., Simmons, K., Lannon, H., Wang, C., Garmsiri, M., Fleet, L., \& Wetsch, L. (2019). Adult learners' perceptions of self-directed learning and digital technology usage in continuing professional education: An update for the digital age. Journal of Adult and Continuing Education, 25(1), 74-93. https://doi.org/10.1177/1477971419827318

Dehghanzadeh, H., Fardanesh, H., Hatami, J., Talaee, E., \& Noroozi, O. (2019). Using gamification to support learning English as a second language: a systematic review. Computer Assisted Language Learning, 0(0), 1-24. https://doi.org/10.1080/09588221.2019.1648298

Demir, M. D., \& Tavil, Z. M. (2021). The effect of technology-based materials on vocational high school students' listening skill. Journal of Language and Linguistic Studies, 17.

Demírbílek, M., \& Yücel, Z. (2011). İngilizce Öğretmenlerinin Bilgisayarın Yabancı Dil Öğretim ve Öğreniminde Kullanımı Hakkındaki Görüşleri. Uludağ Üniversitesi Eğitim Fakültesi Dergisi, 24(1), 217-246. https://doi.org/10.19171/unefd.63432

Ekinci, E., \& Ekinci, M. (2017). Perceptions of EFL Learners about Using Mobile Applications for English Language Learning: A Case Study. International Journal of Language Academy, 5(5), 175-193.

Erenli, K. (2013). The impact of gamification-recommending education scenarios. International Journal of Emerging Technologies in Learning (iJET), 8(2013).

Erkan, H. C. (2020). Digitalization of English Language Teaching in Higher Education: Insights from English Preparatory Classes in Turkish Universities.

Fallas, J., 2012. Prueba de Hipótesis. [ebook] pp.2,48 49. Available at: $<$ https://www.ucipfg.com/Repositorio/MGAP/MGAP-05/BLOQUE-

ACADEMICO/Unidad-2/complementarias/prueba_hipotesis_2012.pdf> [Accessed 23 February 2021].

Forsythe, E. (2017). Pedagogical rationale for flipped learning and digital 
technology in second language acquisition. In Flipped instruction: Breakthroughs in Research and Practice (pp. 116-130). IGI Global.

García, I. (2013). Learning a Language for Free While Translating the Web. Does Duolingo Work? International Journal of English Linguistics, 3(1), 19-25. https://doi.org/10.5539/ijel.v3n1p19

İŞCAN, A. (2011). The Role and Significance of Films in the Turkish Teaching as Foreign Language. Journal of Turkish Studies, Volume 6 Issue 3(6), 939-948. https://doi.org/10.7827/turkishstudies.2692

Karaduman, G. (2018). Bağlantıcılık temelli mikro-öğrenmenin yabancı dil ögrreniminde uygulanabilirliği: Voscreen örneği (Master's thesis, Anadolu Üniversitesi).

Kiliçkaya, F. (2018). Information and Communications Technology (ICT) in Listening Instruction. The TESOL Encyclopedia of English Language Teaching, 1-7. https://doi.org/10.1002/9781118784235.eelt0601

Krumlová, M. (2017). Využití aplikací ke zlepšení poslechových dovedností studentů angličtiny.

Lewandowska, A., \& Jankowski, J. (2017). The negative impact of visual web advertising content on cognitive process: towards quantitative evaluation. International Journal of Human Computer Studies, 108(July 2017), 41-49. https://doi.org/10.1016/j.ijhcs.2017.07.002

Machimbarrena, J. M., Calvete, E., Fernández-González, L., Álvarez-Bardón, A., Álvarez-Fernández, L., \& González-Cabrera, J. (2018). Internet risks: An overview of victimization in cyberbullying, cyber dating abuse, sexting, online grooming and problematic internet use. International journal of environmental research and public health, 15(11), 2471.

Molina-García, P. F., Molina-García, A. R., \& Gentry-Jones, J. (2021). La gamificación como estrategia didáctica para el aprendizaje del idioma inglés. Dominio de las Ciencias, 7(1), 722-730.

Ortiz-Colón, A. M., Jordán, J., \& Agredal, M. (2018). Gamificación en educación: una panorámica sobre el estado de la cuestión. Educação e Pesquisa, 44.

Ozer, O., \& Kılıç, F. (2018). The effect of mobile-assisted language learning 
environment on EFL students' academic achievement, cognitive load and acceptance of mobile learning tools. EURASIA Journal of Mathematics, Science and Technology Education, 14(7), 2915-2928.

Paker, T. (2012). Türkiyeâde Neden Yabancı Dil (İngilizce) Öğretemiyoruz ve Neden Öğrencilerimiz İletişim Kurabilecek Düzeyde İngilizce Öğrenemiyor? Pamukkale Üniversitesi Eğitim Fakültesi Dergisi, 32(32), 89-94-94. https://doi.org/10.9779/PUJE563

Ronquillo Perlaza, L. G. (2016). The application of icts as pedagogical tools to improve the english language teaching-learning process in the second year of baccalaureate students from "MARGARITA CORTÉS" Educational Institution, in the City of Esmeraldas (Doctoral dissertation, Ecuador-PUCESE-Escuela de Lingüística Aplicada).

Samaha, M., \& Hawi, N. S. (2016). . Computers in Human Behavior, 57, 321-325. https://doi.org/10.1016/j.chb.2015.12.045

Sykes, J. M. (2018). Digital games and language teaching and learning. Foreign Language Annals, 51(1), 219-224.

Subrahmanyam, K., Michikyan, M., Clemmons, C., Carrillo, R., Uhls, Y. T., \& Greenfeld, P. M. (2013). Learning from paper, learning from screens: Impact of screen reading and multitasking conditions on reading and writing among college students. International Journal of Cyber Behavior, Psychology and Learning, 3(4), 1-27. https://doi.org/10.4018/ijcbpl.2013100101

Taylan, U. (2018). VoScreen Online Foreign Language Learning Environment. Journal of Educational Technology and Online Learning, 1(1), 60-69. https://doi.org/10.31681/jetol.376754

Tovar-Viera, R. (2020). Examining Technological Pedagogical Content Knowledge Competencies in In-Service Teachers. Veritas \& Research, 1(2), 115-125.

Trémion, V. (2019). Etude de représentations sur les innovations en classe inversée à l'université. Spirale-Revue de recherches en éducation, (1), 11-21.

Türel, Y. K., \& Davudova, E. (2019). USING MOBILE APPLICATIONS IN FOREIGN LANGUAGE LEARNING. дəз

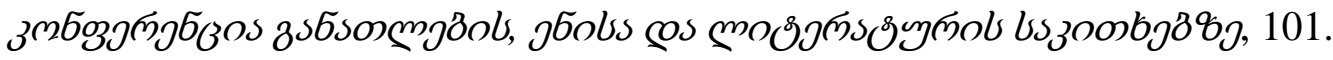


Tütüncü, N., \& Aksu, M. (2018). A systematic review of flipped classroom studies in Turkish education. International Journal of Social Sciences and Education Research, 4(2), 207-229.

Valderrama, B. (2015). Los secretos de la gamificación: 10 motivos para jugar. Capital humano, 295, 73-78.

Vesselinov, R., \& Grego, J. (2012). Duolingo Effectiveness Study. City University of New York, USA, December 2012, 1-25. http://static.duolingo.com/s3/DuolingoReport_Final.pdf\%5Cnpapers3://publication/u uid/66F5DF12-D322-4400-8488-A09AE8EF47F1

Vesselinov, R. \& Grego, J. (2016). The Busuu efficacy study. Londra \& New York: Busuu, 1-30.

Viera, R. T., \& Sánchez, D. I. V. (2020). Research on Technology Competencies in EFL Language Instructors: Technology-Pedagogy-Content in Language Teaching. SCRIPT Journal of Linguistics and English Teaching, 5(1).

Yienger, M. E. (2016). Too Much Tech Harms Reading Retention in Young Children. Inquiries Journal/Student Pulse, 8(03), 1-4. http://www.inquiriesjournal.com/articles/1374/too-much-tech-harms-readingretention-in-young-children 